\title{
Prática de lazer em adolescentes e fatores associados: implicações para o cuidado
}

Leisure practice in adolescents and associated factors: implications for care Práctica de actividades recreativas en adolescentes y factores asociados: consecuencias para el cuidado

Lethícia Farias Marcino ${ }^{1}$ (D) https://orcid.org/0000-0002-1064-2424 Bianca Cristina Ciccone Giacon-Arruda ${ }^{1}$ (i) https://orcid.org/0000-0002-8433-6008

Elen Ferraz Teston ${ }^{1}$ iD https://orcid.org/0000-0001-6835-0574

Albert Schiaveto de Souza ${ }^{1}$ iD https://orcid.org/0000-0003-0017-672x

Priscila Maria Marcheti ${ }^{1}$ id https://orcid.org/0000-0002-1662-4139

Helder de Pádua Lima² io https://orcid.org/0000-0002-3795-6343

Sonia Silva Marcon ${ }^{3}$ iD https://orcid.org/0000-0002-6607-362X

Nathan Aratani ${ }^{1}$ id https://orcid.org/0000-0002-4602-7319

Como citar:

Marcino LF, Giacon-Arruda BC, Teston EF, Souza AS, Marcheti PM, Lima HP, et al. Prática de lazer em adolescentes e fatores associados: implicações para 0 cuidado. Acta Paul Enferm. 2022;35:eAPE02041.

DOI

http://dx.doi.org/10.37689/acta-ape/2022A002041

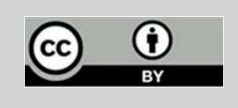

Descritores

Adolescente; Atividades de lazer; Qualidade de vida; Saúde mental

Keywords

Adolescent; Leisure activities; Mental health; Quality of life

Descriptores

Adolescente; Calidad de vida; Actividades recreativas; Salud mental

Submetido 29 de Julho de 2020

Aceito

23 de Março de 202

Autor correspondente Bianca Cristina Ciccone Giacon-Arruda E-mail: biagiacon@gmail.com

Editor Associado (Avaliação pelos pares): Ariane Ferreira Machado Avela (https://orcid.org/0000-0001-7479-8121) Escola Paulista de Enfermagem, Universidade Federal de São Paulo, São Paulo, SP, Brasil

\section{Resumo}

Objetivo: Relacionar a realização de práticas de atividades de lazer com qualidade de vida, bem-estar subjetivo, ansiedade e depressão em adolescentes.

Métodos: Estudo quantitativo, transversal analítico, desenvolvido com 272 adolescentes do ensino médio de três escolas públicas estaduais de uma capital da região centro-oeste. A coleta de dados ocorreu no mês de maio de 2019, mediante autoaplicação de quatro instrumentos: escala de bem-estar subjetivo, escala hospitalar de ansiedade e depressão, questionário de qualidade de vida kidscren-52 e questionário sociodemográfico. A associação entre as variáveis independentes foi verificada por meio do teste t-student e qui-quadrado de fischer.

Resultados: Quase a metade dos adolescentes (49,6\%) realizava alguma atividade de lazer, e nestes foi verificado escores mais baixo para depressão $(p=0,008)$, mais alto nos afetos positivos do bem-estar subjetivo $(p=<0,001)$, e para algumas dimensões da qualidade de vida, tais como saúde e atividade física $(p=<0,001)$, sentimentos ( $p=0,0046)$, estado emocional ( $p=0,033)$, autonomia e tempo livre $(p=0,007)$, aspecto financeiro $(p=0,001)$ e amigos e apoio social $(p=0,002)$. Não houve associação significante entre a prática de atividade de lazer com os escores de ansiedade, afetos negativos e satisfação com a vida.

Conclusão: A prática de atividades de lazer por adolescentes é baixa e associa-se a menores escores para depressão e escores mais altos para algumas dimensões da qualidade de vida e bem-estar subjetivo.

\section{Abstract}

Objective: To relate the performance of leisure activity practices with quality of life, subjective well-being, anxiety, and depression in adolescents.

Methods: This is a quantitative, cross-sectional, analytical study, developed with 272 high school adolescents from three public schools in a center-western capital. Data collection occurred in May 2019 through selfapplication of four instruments: subjective well-being scale, hospital anxiety and depression scale, kidscren-52 quality of life questionnaire and sociodemographic questionnaire. The association among the independent variables was verified by Student's t-test and Fischer's chi-square test.

Results: Almost half of adolescents (49.6\%) performed some leisure activity and it was verified lower scores for depression ( $p=0.008)$, higher in the positive affects of subjective well-being $(p=<0.001)$, and for some dimensions of quality of life, such as health and physical activity $(p=<0.001)$, feelings $(p=0.0046)$, emotional status $(p=0.033)$, autonomy and free time $(p=0.007)$, financial aspect $(p=0.001)$ and friends and social support $(p=0.002)$. There was no significant association between leisure activity and anxiety scores, negative affects and life satisfaction. 
Conclusion: The practice of leisure activities by adolescents is low and is associated with lower scores for depression and higher scores for some dimensions of quality of life and subjective well-being.

\section{Resumen}

Objetivo: Relacionar la práctica de actividades recreativas con calidad de vida, bienestar subjetivo, ansiedad y depresión en adolescentes.

Métodos: Estudio cuantitativo, transversal analítico, llevado a cabo con 272 adolescentes de tres escuelas secundarias públicas regionales de una capital de la región centro-oeste. La recopilación de datos se realizó en el mes de mayo de 2019, mediante la autoaplicación de cuatro instrumentos: escala de bienestar subjetivo, escala hospitalaria de ansiedad y depresión, cuestionario de calidad de vida KIDSCREEN-52 y cuestionario sociodemográfico. La asociación entre las variables independientes se verificó a través del test-T student y ji cuadrado de Fischer.

Resultados: Casi la mitad de los adolescentes (49,6 \%) realizaba alguna actividad recreativa, en los que se verificó puntuación más baja de depresión $(p=0,008)$, más alta en los afectos positivos de bienestar social subjetivo $(p=<0,001)$, y en algunas dimensiones de calidad de vida, tales como salud y actividad física $(p=<0,001)$, sentimientos $(p=0,0046)$, estado emocional $(p=0,033)$, autonomía y tiempo libre $(p=0,007)$, aspecto financiero ( $p=0,001)$ y amigos y apoyo social $(\mathrm{p}=0,002)$. No hubo relación significativa entre la práctica de actividades recreativas y la puntuación de ansiedad, afectos negativos y satisfacción con la vida.

Conclusión: La práctica de actividades recreativas de adolescentes es baja y se relaciona con una menor puntuación de depresión y una mayor puntuación en algunas dimensiones de la calidad de vida y el bienestar subjetivo.

\section{Introdução}

A adolescência é uma fase marcada pela associação de fatores individuais, coletivos, familiares, sociais, políticos e econômicos, que impactam diretamente na produção de saúde dessa população. ${ }^{(1)}$ Assim, quanto maior a exposição à fatores de risco e situaçôes de vulnerabilidade, maior a possibilidade de adoecimento mental e de interferência no desenvolvimento individual, nas atitudes, nos comportamentos e nas relaçôes sociais na vida adulta. ${ }^{(1-3)}$

Em contrapartida, fatores, como a autonomia, a autoestima, a religiosidade e espiritualidade, o lazer e o bem-estar subjetivo, o apoio familiar e social, o otimismo e a criatividade atuam como protetores da saúde mental da população jovem por envolverem sentimentos positivos, maior satisfação e melhor qualidade de vida. ${ }^{(4,5)}$

Em específico, tem sido identificado que o lazer, compreendido como um conjunto de atividades realizadas no tempo livre, ou seja, após as obrigaçóes relacionadas ao trabalho ou estudo, é considerado uma forma de descanso e recreação, e, por conseguinte, proporciona bem-estar, qualidade de vida e saúde. ${ }^{(6)}$

O desenvolvimento de atividades de arte e da prática de exercícios físicos, como atividade de lazer, apresenta associação com maiores escores de qualidade de vida, bem-estar subjetivo e satisfação com a vida. ${ }^{(4,5)}$ Ademais, prática de esportes influenciam diretamente no desenvolvimento de uma vida saudável, principalmente a longo prazo, com hábitos de exercício físico na vida adulta. ${ }^{(7,8)}$ Além disso, consti- tui também uma estratégia para estabelecimento do convívio social o que contribui para a saúde mental. ${ }^{(9)}$

Embora os benefícios das atividades de lazer na saúde física e mental seja consenso na literatura, observou-se escassez de estudos que explorem a associação entre a prática de lazer com a qualidade de vida, bem-estar subjetivo e o adoecimento mental em adolescentes.

Acredita-se que, aprofundar o conhecimento sobre esse tema pode contribuir para a elaboração, implantaçáo e implementação de açóes educativas pelos enfermeiros e, no contexto macro, de políticas públicas de promoção de saúde e prevenção de agravos direcionadas a população adolescente, principalmente em relação à saúde mental. Assim, o presente estudo objetivou relacionar a realização de práticas de atividades de lazer com qualidade de vida, bem-estar subjetivo, ansiedade e depressão em adolescentes.

\section{Métodos}

Estudo quantitativo, transversal analítico, realizado em três escolas públicas estaduais da capital do Estado de Mato Grosso do Sul, selecionadas por conveniência por constituírem campo de atuação da universidade e por se localizarem em uma regiáo de elevada vulnerabilidade social.

Em relação às características das três escolas, todas ofertavam turmas de alunos de ensino médio nos três períodos, constituídas: 1) sete turmas e média de 230 alunos matriculados; 2) 22 turmas 
e média de 640 alunos matriculados; 3) 14 turmas com média de 520 alunos matriculados. Todas as escolas ofereciam atividades complementares, como lutas, ginástica, jogos esportivos e aulas de música.

Para seleção dos participantes, adotou-se a amostragem não probabilística e os seguintes critérios de inclusão: ter entre 15 e 18 anos e estar regularmente matriculados no ensino médio em uma das três escolas no período diurno (matutino e vespertino). Por sua vez, constituíram critérios de exclusão: ausência nos dias destinados à coleta de dados e capacidade cognitiva alterada devido efeitos de álcool ou drogas, ou a alguma condição de saúde.

Os dados foram coletados mediante autoaplicação de quatro instrumentos ocorreu no mês de maio de 2019, durante o turno escolar, em único dia e horário definido pela coordenação da escola de modo a interferir o mínimo possível nas atividades de ensino. O tempo médio para o preenchimento pelas turmas foi de 40 minutos.

Os procedimentos para a coleta de dados envolveram: a) reuniáo com os diretores das escolas que se encarregaram de apresentar a proposta aos professores; b) reuniáo com os adolescentes para apresentaçáo dos objetivos do estudo e o tipo de participação desejada. Para todos os que se interessaram, foi entregue o Termo de Consentimento Livre e Esclarecido (TCLE), e o Termo de Assentimento Livre e Esclarecido (TALE) para os menores de 18 anos, junto ao TCLE para os responsáveis.

Dentre o total de 332 alunos que atenderam aos critérios de inclusão e foram convidados, três foram excluídos em decorrência de comprometimento cognitivo no momento da coleta de dados devido ao uso de álcool e outras drogas, e 57 não estavam presentes no dia da coleta ou náo apresentaram os termos de consentimento/assentimento assinados.

Os quatro instrumentos utilizados na coleta de dados foram:

A. Questionário de caracterização socioeconômica do adolescente, elaborado pelos autores, contendo: sexo, data de nascimento/idade, cor, naturalidade, ano de escolaridade, trabalho, com quem reside, estado civil, filhos, e prática de atividade de lazer.

B. Escala de Bem-Estar Subjetivo (EBES) validada no Brasil constituída por 62 itens divididos em três sub-escalas, sendo 21 de afeto positivo, 26 de afeto negativo e 15 de satisfação com a vida. As respostas são apresentadas em escala do tipo Likert de cinco pontos (que varia de 1 [nem um pouco] a 5 [extremamente]). ${ }^{(10)}$ Para análise o total de pontos alcançado por cada indivíduo foi dividido pelo número de questóes, sendo considerado escore alto quando igual ou maior que três e baixo quando inferior a três, nas três sub-escalas.

C. Escala Hospitalar de Ansiedade e Depressão (HAD), composta por 14 itens (sete que avaliam ansiedade e sete a depressão). As respostas são apresentadas em escala do tipo Likert de 4 pontos (zero a três), com pontuação máxima em cada sub-escla de 21 pontos. ${ }^{(11)} \mathrm{A}$ análise é feita em cada escala sub-escala, sendo considerado os seguintes intervalos para avaliação dos níveis de ansiedade e depressão: 0 a 7: improvável; 8 a 11: questionável/ duvidoso; 12 a 21: provável. Por mais que a HAD indique em seu título o ambiente hospitalar, outros estudos já a utilizaram na população geral (adolescentes, adulto e idosos) e em outros contextos, indicando a sua aplicabilidade. ${ }^{(12,13)}$

D. Questionário de Qualidade de Vida Kidscreen 52 , constituído por 52 questóes, direcionadas à percepção de dez dimensôes de qualidade de vida. É uma escala de resposta tipo Likert de um a cinco pontos para avaliar cada dimensão e, quanto maior o escore, maior a qualidade de vida. ${ }^{(14)}$

Os dados foram digitalizados em dupla entrada e organizados em planilhas do Excel do Microsoft Office Professional Plus ${ }^{\mathrm{R}} 2016$ e posteriormente analisados por meio de estatística descritiva e inferencial, com os testes t-student e qui-quadrado no Statistical Package for the Social Sciences (SPSS), versão 23.0, considerando um nível de significância de 5\%.

Esse estudo está inserido em um estudo maior intitulado "Saúde mental, qualidade de vida e bem-estar subjetivo de adolescentes do ensino médio." o qual foi aprovado pelo Comitê de Ética em Pesquisa com Seres Humanos da Universidade Federal de Mato Grosso do Sul, sob parecer no. 3.231.242 (CAAE: 07702619.2.0000.0021), respeitando todas as normas nacionais e internacionais de ética em pesquisa com seres humanos. 


\section{Resultados}

Participaram do estudo 272 alunos, a maioria do sexo feminino $(62,5 \%)$, com idade entre 15 e 18 anos, solteiros $(96,3 \%)$ e de cor parda $(64,3 \%)$. Em relação com quem reside, $45,2 \%$ moravam com o pai e a mãe, $23,5 \%$ com o pai ou a mãe e $30,5 \%$ com outros (tio, tia, avó, avô, entre outros). Do total, $0,7 \%$ referiram ter filhos, $16,1 \%$ realizavam atividades de trabalho remunerado, e $6,3 \%$ possuíam alguma doença, deficiência e/ou problema médico crônico, sendo a doença respiratória a mais relatada. Náo houve discrepância em relaçáo a quantidade de alunos por ano escolar do primeiro ao terceiro, sendo $34,9 \%, 36,4 \%$ e $28,7 \%$ respectivamente.

A prática de lazer foi referida por $49,6 \%$, sendo citadas atividades físicas ou esporte $(78,5 \%)$ como prática de luta, academia, caminhada, futebol, vôlei, ginástica e andar de bicicleta; atividades eletrônicas (17\%) que envolviam mexer no celular, assistir vídeos, televisão, séries e jogos; atividades culturais (14\%) como leitura, dança, ouvir música, tocar instrumento musical, visitar locais da cidade, estudar e realizar cursos; passeio com amigos e família, no shopping, tomar "tereré" (bebida típica do estado) e ir à casa da família $(7,4 \%)$. Ademais, 7,4\% dos participantes indicaram outras atividades e 1,5\% não definiu.

Observa-se na tabela 1 que indivíduos que praticam alguma atividade de lazer tem escores mais baixo para depressão $(\mathrm{p}=0,008)$ e mais alto para afetos positivos $(\mathrm{p}=<0,001)$.

$\mathrm{Na}$ figura 1, observa-se que adolescentes que praticam atividade de lazer tem escores mais alto para qualidade de vida nas dimensóes saúde e atividade física $(\mathrm{p}=<0,001)$, sentimentos $(\mathrm{p}=0,046)$, estado emocional $(\mathrm{p}=0,033)$, autonomia e tempo livre $(\mathrm{p}=0,007)$, aspecto financeiro $(\mathrm{p}=0,001)$ e amigos e apoio social $(\mathrm{p}=0,002)$.

A tabela 2, apresenta os resultados da avaliaçáo da associação entre a prática de atividade de lazer e a classificaçáo dos alunos em relação ao sexo, à depressão, ansiedade e fatores do bem-estar subjetivo.

Não houve associação significante entre a prática de atividade de lazer com a ansiedade, afetos negativos e satisfação com a vida.
Tabela 1. Associação entre a prática de atividade de lazer e os escores de ansiedade, depressão, e de bem-estar subjetivo

\begin{tabular}{|c|c|c|c|c|c|}
\hline \multirow{3}{*}{ Variáveis } & \multicolumn{4}{|c|}{ Prática de atividade de lazer } & \multirow{3}{*}{$p$-value } \\
\hline & \multicolumn{2}{|r|}{ Sim } & \multicolumn{2}{|c|}{ Não } & \\
\hline & $\mathrm{n}$ & $Y^{\star *}$ & $\mathrm{n}$ & $Y^{\star \star}$ & \\
\hline \multicolumn{6}{|l|}{ Ansiedade e depressão } \\
\hline Ansiedade & 134 & $8,84 \pm 0,39$ & 130 & $9,39 \pm 0,41$ & 0,328 \\
\hline Depressão & 134 & $7,32 \pm 0,34$ & 130 & $8,62 \pm 0,35$ & 0,008 \\
\hline \multicolumn{6}{|l|}{ Bem-estar subjetivo } \\
\hline Afetos positivos & 124 & $2,98 \pm 0,07$ & 124 & $2,54 \pm 0,06$ & $<0,001$ \\
\hline Afetos negativos & 125 & $2,58 \pm 0,07$ & 121 & $2,56 \pm 0,07$ & 0,831 \\
\hline Satisfação de vida & 120 & $3,09 \pm 0,03$ & 121 & $3,05 \pm 0,03$ & 0,752 \\
\hline
\end{tabular}

$\mathrm{X}^{\star}$ - Média; $Y^{\star \star}$ - Erro padrão; P-valor ${ }^{\star \star \star}$ - Significância referente ao teste de t- student

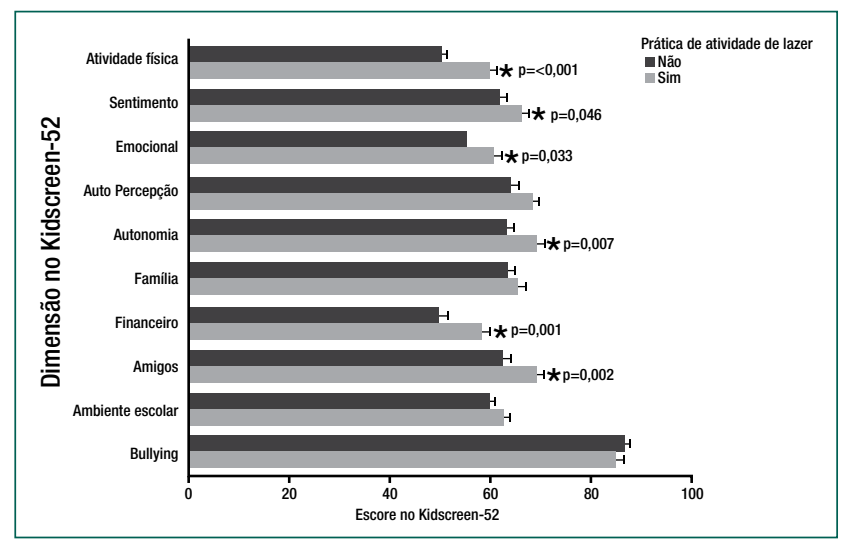

0s resultados estão apresentados em média \pm erro padrão da média. $p$-value no teste t-student

Figura 1. Associação entre a prática de atividade de lazer e qualidade de vida

Tabela 2. Associação entre a prática de atividade de lazer com o sexo, a depressão, ansiedade e bem-estar subjetivo

\begin{tabular}{|c|c|c|c|}
\hline \multirow{2}{*}{ Variável } & \multicolumn{2}{|c|}{$\begin{array}{l}\text { Prática de atividade de } \\
\text { lazer }\end{array}$} & \multirow{2}{*}{$p$-value ${ }^{\star}$} \\
\hline & $\underset{\mathrm{n}(\%)}{\operatorname{Sim}}$ & $\begin{array}{l}\text { Não } \\
\text { n(\%) }\end{array}$ & \\
\hline \multicolumn{4}{|l|}{ Sexo $(n=267)$} \\
\hline Masculino & $61(60,4)$ & $40(39,6)$ & 0,009 \\
\hline Feminino & $73(44,0)$ & $93(56,0)$ & \\
\hline \multicolumn{4}{|c|}{ Depressão (n=264) } \\
\hline Sem & $86(58,1)$ & $62(41,9)$ & 0,007 \\
\hline Com & $48(41,4)$ & $68(58,6)$ & \\
\hline \multicolumn{4}{|c|}{ Ansiedade $(n=264)$} \\
\hline Sem & $63(52,5)$ & $57(47,5)$ & 0,605 \\
\hline Com & $71(49,3)$ & $73(50,7)$ & \\
\hline \multicolumn{4}{|c|}{ Bem-estar subjetivo } \\
\hline \multicolumn{4}{|c|}{ Afetos positivos ( $\mathrm{n}=248$ ) } \\
\hline Baixa & $59(40,4)$ & $87(59,6)$ & 0,001 \\
\hline Alto & $65(63,7)$ & $37(36,3)$ & \\
\hline \multicolumn{4}{|c|}{ Afetos negativos ( $n=246)$} \\
\hline Baixo & $80(47,6)$ & $88(52,4)$ & 0,141 \\
\hline Alto & $45(57,7)$ & $33(42,3)$ & \\
\hline \multicolumn{4}{|c|}{ Satisfação de vida ( $\mathrm{n}=241)$} \\
\hline Baixo & $46(51,1)$ & $44(48,2)$ & 0,752 \\
\hline Alto & $74(49,0)$ & $77(51,0)$ & \\
\hline
\end{tabular}

0s resultados estão apresentados em frequência relativa (frequência absoluta); $p$-value $e^{\star \star \star}=$ Significância referente ao $p$-value no teste do qui-quadrado 


\section{Discussão}

A limitação do estudo refere-se à composição intencional da amostra de adolescentes em estudo, visto que os mesmos foram identificados a partir de três escolas localizadas na mesma região do município, a qual é caracterizada pelo baixo poder socioeconômico. Assim, a realização de novos estudos com adolescentes residentes em outras áreas com características socioeconômicas, territoriais e com maior investimento em políticas públicas de esporte e lazer tornam-se importantes.

Esse estudo oferece subsídios para o planejamento de açóes de saúde que incentivem a prática de atividades de lazer por adolescentes, em especial a prática de atividade física, considerando-a como fator de proteção à saúde mental, que favorece positivamente a qualidade de vida e o bem-estar. Ademais, oportuniza-se a partir dos resultados a reflexão em relação a necessidade de políticas públicas além da área da saúde, como de esporte e lazer, que possibilitem o acesso, a motivação e o incentivo à essa população.

Os resultados indicaram que quase metade dos participantes referiu realizar atividade de lazer, sendo a atividade física a mais referida. Contudo, a literatura aponta que a prática de atividade física pelos adolescentes é baixa. ${ }^{(2,9,15,16)}$ Um estudo realizado com dados de 298 pesquisas em escolas de 146 diferentes países, totalizando uma amostra de 1,6 milhóes de alunos na faixa etária de 11 a 17 anos, demonstrou que $81 \%$ dessa população estudada realizavam atividade física de forma insuficiente quando comparada com as recomendaçôes da OMS. ${ }^{(9)} \mathrm{O}$ que suscita a necessidade de açôes intersetoriais que favoreçam a motivação dos adolescentes a realização destas, especialmente porque, incentivos nessa fase do desenvolvimento tem apontado resultados positivos na mudança de comportamento também na vida adulta. ${ }^{(9-17)}$

Considerando este aspecto, o Plano de Ação Global de Atividade Física defende a importância na realização desta para prevenir e tratar doenças não transmissíveis, além de melhorar a saúde mental, qualidade de vida e bem-estar. Ressalta ainda que, a atividade física e de lazer, como jogos e recreação, são importantes em todas as idades. E, na infância e adolescência, contribui para um crescimento e desenvolvimento saudável. ${ }^{(2)}$

Estudo realizado em Minas Gerais, verificou que $35,5 \%$ dos jovens que participaram de um Programa de Esporte e Lazer relataram menos doenças do que aqueles $(50,9 \%)$ que não participaram. ${ }^{(18)}$ Ainda nesta direção, estudo que avaliou 72 adolescentes do ensino básico, constatou que a realização de atividade física pelo menos três vezes na semana melhora as "aptidôes cardiorrespiratórias e cardiovasculares, além de proporcionar diversos benefícios físicos, psicológicos e sociais". ${ }^{(19)}$

A associação da prática de atividade física com maiores escores de afetos positivos, que incluem alegria, entusiasmo, orgulho e felicidade, reitera a importância do estímulo a prática dessas atividades para esse público, em especial pelo fato destes afetos implicarem diretamente na saúde mental. Ademais, na adolescência, os afetos são vivenciados com maior intensidade devido as mudanças biopsicossociais ocorridas nesse período, e as emoções irão refletir no bem-estar e, consequentemente, na qualidade e satisfação com a vida. ${ }^{(20)}$

A prática de atividades de lazer, por sua vez, influencia positivamente na qualidade de vida e bem-estar subjetivo. Isso porque ela propicia divertimento e oferece oportunidades de relaçóes sociais e aprimoramento pessoal, favorecendo a melhora da cognição, da autoestima, de sentimentos de bem-estar e socialização. Ademais, a associação destes fatores atua como coadjuvante na contençáo de fatores de risco para o adoecimento mental e físico. ${ }^{(18)}$

O bem-estar subjetivo elevado na adolescência contribui, também, para uma vida adulta saudável, física e mentalmente. E, com isso, em maiores chances do indivíduo controlar suas emoçôes e de desenvolver açóes de autocuidado. ${ }^{(21)}$

Posto isso, reitera-se que a qualidade de vida e o bem-estar subjetivo possuem características que podem proporcionar mudanças no estilo de vida dos adolescentes, principalmente no aspecto psicossocial, ao promover sentimentos positivos e hábitos de vida saudáveis, os quais funcionam como fator de proteção. Por outro lado, a baixa qualidade de vida, de afetos positivos e de bem-estar relacionam-se a níveis mais altos de depressão e ansiedade. ${ }^{(22)}$ 
Nesse sentido, além do incentivo para realização de exercícios de lazer, como atividade física, outros fatores necessitam ser considerados no planejamento de ações estratégicas voltadas aos adolescentes como, por exemplo, a desmotivação, organização do tempo, falta de companhia, acúmulo de tarefas diárias, falta de incentivo em casa pelos familiares, dificuldade de deslocamento, ausência de locais adequados e gratuitos, e de segurança. ${ }^{(8,16,23)}$

Ademais, observou-se que adolescentes que praticam alguma atividade de lazer apresentaram escores mais baixos para depressão. Cabe salientar que esta doença, gera inúmeros riscos à saúde mental dos adolescentes, desde o aumento na dificuldade de interação social, do distanciamento, rejeição de seus pares e sentimento de solidão, até aqueles mais graves, como suicídio. ${ }^{(24)}$ Além disso, no contexto de vida geral, a depressão influencia negativamente na qualidade de vida e no bem-estar. ${ }^{(22)}$

Estudo de metanálise demonstrou que, independentemente da localidade de moradia ou idade, a atividade física funciona como um fator de prevenção a depressão, bem como na redução dos sintomas em indivíduos deprimidos. ${ }^{(25)}$ Do mesmo modo, estudo realizado com 269 indivíduos adultos da cidade Karachi, no Paquistão, apontou associação entre a prática de atividade física e menor frequência de ansiedade e depressão. ${ }^{(26)}$

Em Campo Grande - MS, estudo identificou que a implantação/criação de áreas verdes contribui de forma positiva nos aspectos fisiológicos e psicológicos da população. Isso porque, nestes locais são implementadas açôes e atividades, individuais e coletivas, de promoção à saúde, além de favorecer a relação harmônica entre o homem e a natureza. Os autores concluíram que estes espaços proporcionam melhor qualidade de vida, bem-estar e lazer a população local e aos visitantes. ${ }^{(27)}$

Cabe destacar que a elevada prevalência de fatores de risco para doenças cardiovasculares em universitários, como o tabagismo, consumo de bebidas alcoólicas, sedentarismo, alimentação desequilibrada e excesso de peso, evidenciam um alerta, pois boa parte destes hábitos tende a ser consolidado na vida adulta, contribuindo para o desenvolvimento de doenças crônicas cada vez mais cedo. ${ }^{(28)}$
Os resultados dos estudos supracitados reforçam a importância de políticas públicas direcionadas aos adolescentes, em especial aquelas que favoreçam o acesso a ações de lazer. A promoção de ações educativas em grupo e de promoção da saúde devem ser iniciadas o mais precoce possível, para que os indivíduos tenham maior aderência a hábitos saudáveis. Ademais, a consulta de enfermagem direcionada à essa público necessita atribuir enfoque especial em relação a esses aspectos, principalmente pelo fato dos hábitos inadequados de vida influenciarem na autoimagem e por conseguinte na saúde mental.

No contexto das Políticas Públicas intersetoriais (educação e saúde), o Programa Saúde na Escola (PSE), constitui uma ferramenta de grande valia para a elaboração de estratégias conjuntas que favoreçam a motivação e ao reconhecimento da necessidade de mudança nos estilos de vida, como por exemplo na realização de atividade física. ${ }^{(29)}$ Ademais, açóes que favoreçam a promoção da saúde mental e que reforcem o reconhecimento de fatores de proteçáo para uma boa qualidade de vida e afetos positivos necessitam ser planejadas e implementadas continuamente. Desse modo, considerando que a enfermagem, juntamente com a equipe de saúde da Família, tem a possibilidade de desenvolver açôes em ambientes extra muros, destaca-se a oportunidade de captar e atrair a população jovem para os serviços de promoção e prevenção na Unidade de Saúde.

Frente a isso, a enfermagem tem papel fundamental na ocupação dos diferentes espaços sociais com vistas a discussão frente a implementação das políticas públicas. Bem como, na articulação entre os diferentes setores, como saúde e educação. O profissional de enfermagem, conhecendo as políticas, as açóes, a vulnerabilidade pelo qual esse adolescente está exposto e as necessidades, tem um importante papel no cumprimento dessas açóes e acompanhamento da saúde mental dos adolescentes, principalmente na APS. ${ }^{(1,30)}$ Além disso, por ocupar espaços de representação em diferentes recursos sociais, necessita problematizar as questôes relacionadas aos serviços ofertados e as demandas específicas dessa população.

No entanto, alguns fatores dificultam a implementação dessas açóes, como a baixa adesão dos adolescentes, que raramente procuram a unidade 
básica de saúde, a falta de capacitação específica para abordar e atuar junto a esse público, bem como recursos suficientes e que atraiam a atenção e estratégias/abordagens que favoreçam o protagonismo dos adolescentes. ${ }^{(31)}$

É importante, também, que o enfermeiro desenvolva competências e práticas de cuidado, considerando os diferentes contextos e determinantes que possam estar associados ao processo de saúde-doença, re-signifique as concepçóes de adolescência, desenvolva uma escuta sensível e acessível, e ações que possibilitem o adolescente ser protagonista no seu processo de saúde doença. ${ }^{(1,30)}$

Destarte, é necessário repensar os processos de trabalhos dos profissionais e equipes de saúde da atenção básica, no cuidado ao adolescente, buscando fortalecer as parcerias intersetoriais, além de propor açóes no território e não apenas concentradas nos equipamentos de saúde, ${ }^{(32)}$ visto que ele é um dos atores principais na articulação entre os setores de saúde e educação, contextos importantes na promoção e prevenção de saúde. Por fim, um aspecto que não pode ser esquecido é o envolvimento, tanto quanto possível, da família e dos amigos ou pelo menos, que se considere o contexto familiar nas proposiçóes. ${ }^{(33)}$

Portanto, esse estudo demonstrou a importância de aplicar as políticas públicas e acompanhar esse público mais de perto, visando não apenas a sua saúde física, mas, também, a saúde mental. E, acredita-se que poderá servir de subsídio para novas pesquisas a respeito da temática abordada.

\section{Conclusão}

Identificou-se que mais da metade dos adolescentes em estudo não realizam atividades de lazer, cuja prática apresentou associação com melhor qualidade de vida e bem-estar subjetivo e menores escores de depressão.

\section{Colaborações}

Marcino LF, Giacon-Arruda BCC, Teston EF, Souza AS, Marcheti PM, Lima HP, Marcon SS, Aratani N contribuíram com a concepção e projeto ou análise e interpretação dos dados, redação do artigo ou revisão crítica relevante do conteúdo intelectual, aprovação final da versão a ser publicada.

\section{Agradecimentos}

O presente trabalho foi realizado com apoio da Fundação Universidade Federal de Mato Grosso do Sul -UFMS/MEC - Brasil, e com apoio da Coordenação de Aperfeiçoamento de Pessoal de Nível Superior Brasil (CAPES) - Código de Financiamento 001.

\section{Referências}

1. Gasparetto AS, Bonfim TA, Teston EF, Marcheti PM, Galera SA, Giacon-Arruda $\mathrm{BC}$. Contexts of vulnerabilities experienced by adolescents: challenges to public policies. Rev Bras Enferm. 2020;73(Suppl 4):e 20190224.

2. World Health Organization. Global action plan on physical activity 2018-2030: more active people for a healthier world. Geneva: WHO; 2018 [cited 2021 Mar 10]. Available from: https://www.who.int/ncds/ prevention/physical-activity/global-action-plan-2018-2030/en/

3. Zinn ME, Huntley ED, Keating DP. Resilience in adolescence: Prospective Self moderates the association of early life adversity with externalizing problems. J Adolesc. 2020;81:61-72.

4. Lima RF, Morais NA. Subjective well-being of children and adolescents: integrative review. Cienc Psicol. 2018;12(2):249-60.

5. Otto C, Haller AC, Klasen F, Hölling H, Bullinger M, Ravens-Sieberer U; BELLA study group. Risk and protective factors of health-related quality of life in children and adolescents: results of the longitudinal BELLA study. PLoS One. 2017;12(12):e0190363.

6. Beserra EP, Sousa LB, Alves MD, Gubert FA. Percepção de adolescentes acerca de suas atividades de vida, trabalho e lazer. Rev Enfermagem UERJ. 2015;23(5):627-32.

7. Jalali-Farahani S, Amiri P, Torshizi K, Cheraghi L, AvatefFazeli M, Azizi F. Association of leisure and occupational physical activities and healthrelated quality of life: Tehran Lipid and Gluycose Study. Health Qual Life Outcomes. 2020;18(1):13.

8. Guthold R, Stevens GA, Riley LM, Bull FC. Global trends in insufficient physical activity among adolescents: a pooled analysis of 298 population-based surveys with 1.6 million participants. Lancet Child Adolesc Health. 2020;4(1):23-35.

9. Shah P, Southerland JL, Slawson DL. Social Support for Physical Activity for High Schoolers in Rural Southern Appalachia. South Med J. 2019;112(12):626-33.

10. Albuquerque AS, Tróccoli BT. Desenvolvimento de uma escala de BemEstar Subjetivo. Psic Teoria Pesq. 2004;20(2):153-64

11. Botega NJ, Bio MR, Zomignani MA, Junior CG, Pereira WA. Transtornos do humor em enfermaria de clínica médica e validação de escala de medida (HAD) de ansiedade e depressão. Rev Saúde Pública. 1995;29(5):355-63. 
12. Barbosa LN, Asfora GC, Moura MC. Anxiety and depression and psychoactive substance abuse in university students. Rev Eletr Saude Mental Alcool Drog. 2020;16(1):1-8.

13. Ribeiro RP, Marziale MH, Martins JT, Ribeiro PH, Robazzi ML, Dalmas JC. Prevalence of Metabolic Syndrome among nursing personnel and its association with occupational stress, anxiety and depression. Rev Lat Am Enfermagem. 2015;23(3):435-40.

14. Guedes DP, Guedes JE. Translation, cross-cultural adaptation and psychometric properties of the kidscreen-52 for the brazilian population. Rev Paul Pediatr. 2011;29(3):364-71.

15. Cureau FV, Silva TL, Bloch KV, Fujimori E, Belfort DR, Carvalho KM, et al. ERICA: leisure-time physical inactivity in Brazilian adolescents. Rev Saúde Pública. 2016;50(Suppl 1):4s.

16. Silva J, Andrade A, Capistrano R, Lisboa T, Andrade RD, Felden EP, et al. Níveis insuficientes de atividade física de adolescentes associados a fatores sociodemográficos, ambientais e escolares. Ciênc Saúde Coletiva. 2018;23(12):4277-88.

17. Corepal R, Tully MA, Kee F, Miller SJ, Hunter RF. Behavioural incentive interventions for health behaviour change in young people (5-18 years old): a systematic review and meta-analysis. Prev Med. 2018;110:55-66.

18. Tolocka RE, Ramos EP, Peruchi LP. Saúde e atividades de lazer de jovens no ensino médio. Rev Aten Saúde. 2019;17(59):39-43.

19. Zawadzki D, Stiegler NF, Brasilio FF. Aptidão e atividade física relacionados à saúde de adolescentes entre 11 e 14 anos. Rev Bras Presc Fisiol Exerc. 2019;13(83):444-53.

20. Oliveira AJ, Sena AC, Martins AG. 0 bem-estar subjetivo em adolescentes: afetos positivos e afetos negativos. Rev Eletr Estácio Papirus. 2018;5(1):69-82.

21. Silva GD, Dell'Aglio DD. Avaliação do bem-estar subjetivo em adolescentes: relações com sexo e faixa etária. Análise Psicológica. 2018;36(2):133-43.

22. Pinto AV, Cavalcanti JG, Araújo LS, Coutinho ML, Coutinho MP. Depressão e adolescência: relação com qualidade de vida e bem-estar subjetivo. Rev Psicologia IMED. 2018;10(2):6-21.
23. Dias DF, Loch MR, Roque RR. Perceived barriers to leisure-time physical activity and associated factors in adolescents. Ciênc Saúde Coletiva. 2015;20(11):3339-50.

24. Grolli V, Wagner FM, Dalbosco SN. Sintomas depressivos e de ansiedade em adolescentes do ensino médio. Rev Psicologia IMED. 2017;(1):87-103.

25. Schuch FB, Vancampfort D, Firth J, Rosenbaum S, Ward PB, Silva ES, et al. Physical Activity and Incident Depression: A Meta-Analysis of Prospective Cohort Studies. Am J Psychiatry. 2018;175(7):63148.

26. Khanzada FJ, Soomro N, Khan SZ. Association of physical exercise on anxiety and depression amongst adults. J Coll Physicians Surg Pak. 2015;25(7):546-8.

27. Melo MR, Bega LR, Taveira NM, Mattos AB. Parque das Nações Indígenas: área de interesse turístico, qualidade de vida e lazer na cidade de Campo Grande - MS. Rev Turismo Contemporâneo. 2015;3(2):299-317.

28. Back IR, Dias BC, Batista VC, Ruiz AG, Peruzzo HE, Druciak CA, et al. Fatores de risco para doenças cardiovasculares em universitários: diferenças entre os sexos. Ciênc Cuid Saúde. 2019;18(1):e40096.

29. Lopes IE, Nogueira JA, Rocha DG. Eixos de ação do Programa Saúde na Escola e Promoção da Saúde: revisão integrativa. Saúde Debate. 2018;42(118):773-89.

30. Santos JS, Andrade RD, Silva MAl, Mello DF. Nurse to adolescent health communication process: approach to Event History Calendar. Rev Bras Enferm. 2020;73(3):e20180454.

31. Vinagre MG, Barros L. Preferências dos adolescentes sobre os cuidados de saúde. Ciênc Saúde Coletiva. 2019;24(5):627-36.

32. Barreto RM, Cavalcante AS, Mira QL, Vasconcelos MI, Brito MC. Ações educativas em saúde para o público adolescente: uma revisão integrativa. Rev APS. 2016;19(2):277-85.

33. Lisboa T, Silva WR, Alexandre JM, Beltrame TS. Suporte social da família e amigos para a prática de atividade física de adolescentes: uma revisão sistemática. Cad Saúde Coletiva. 2018;26(4):351-9. 